\title{
A Statistical Analysis of Intensities Estimation on the Modeling of Non-Life Insurance Claim Counting Process
}

\author{
Uraiwan Jaroengeratikun, Winai Bodhisuwan, Ampai Thongteeraparp \\ Department of Statistics, Kasetsart University, Bangkok, Thailand \\ Email: ur_jaroen@yahoo.com, \{fsciwnb, fsciamu\}@ku.ac.th
}

Received October 1, 2011; revised November 19, 2011; accepted November 28, 2011

\begin{abstract}
This study presents an estimation approach to non-life insurance claim counts relating to a specified time. The objective of this study is to estimate the parameters in non-life insurance claim counting process, including the homogeneous Poisson process (HPP) and the non-homogeneous Poisson process (NHPP) with a bell-shaped intensity. We use the estimating function, the zero mean martingale (ZMM) as a procedure of parameter estimation in the insurance claim counting process. Then, $\Lambda(t)$, the compensator of $N(t)$ is proposed for the number of claims in the time interval $(0, t]$. We present situations through a simulation study of both processes on the time interval $(0, t]$. Some examples of the situations in the simulation study are depicted by a sample path relating $N(t)$ to its compensator $\Lambda(t)$. In addition, an example of the claim counting process illustrates the result of the compensator estimate misspecification.
\end{abstract}

Keywords: Estimating Function; Zero Mean Martingale; Non-Life Insurance Claim Counting Process; Poisson Process; Bell-Shaped Intensity

\section{Introduction}

Nowadays, insurance is a common way of managing risks and the insurance industry has grown rapidly over time. Insurance industry owners, especially, consider the components of risk management, such as the premiums which are the main income of insurance businesses, reserves, underwriting, investment planning, reinsurance planning, etc. Also, estimating claims play an important part in each component in the non-life insurance field. In the past four decades, a few researchers have studied the claim counts model for non-life insurance. Klugman et al. [1] and Denuit et al. [2] were interested in studying the frequency distribution of insurance claims, including the parameter estimation methods. Bühlmann [3,4] presented the credibility approach in the form of a linear function to estimate and predict the expected claim counts in upcoming periods, using past experience of claims as a risk class or related risk classes. Bühlmann's credibility approach is interesting and can be extended to other approaches, such as the Bühlmann-Straub model, Jewell's model or the Exact credibility approach, etc., (see Klugman et al. [1]). Calculating the expected claim counts using the credibility approach only depends on the information from prior experience of claim counts, and does not consider the occurrence behavior of claim counts over time. Some authors have found an alternative approach to claim counts relating to a specified time or their behavior over time, for example, Mikosch [5] viewed the claim counting process as a homogeneous Poisson process (HPP) in the Cramér-Lundberg model, one of the most popular and useful risk models in non-life insurance, and Matsui and Mikosch [6] also considered a Poisson cluster model for the modeling of a total claims amount by a point of claim counts as an HPP with a constant rate of occurrence called the constant intensity. For some non-life insurance portfolios, the claim counts during a time period are caused by periodic phenomena or seasonality. These claim counts are modeled in terms of a non-homogeneous Poisson process (NHPP) with a period time-dependent intensity rate. Morales [7] presented the periodic risk model consisting of the claim counting process with a bell-shaped intensity function (called the Gaussian intensity) of the form

$\lambda(t)=\frac{\lambda^{*}}{\left(\Phi\left(\frac{1}{2 \sigma}\right)-\Phi\left(-\frac{1}{2 \sigma}\right)\right) \sigma \sqrt{2 \pi}} \exp \left\{-\frac{1}{2 \sigma^{2}}\left(t-\frac{1}{2}\right)^{2}\right\} ;$ $t \in[0,1), \lambda(s+t)=\lambda(t)$, where $s$ is an initial season, $s$ $=0,1,2, \cdots, \sigma$ and $\lambda^{*}$ are parameters, and $\Phi$ is the standard normal distribution function. He estimated the unknown parameters of the periodic model intensity by using the maximum likelihood estimation (MLE), and he 
also considered evaluating the ruin probability through a simulation study. Furthermore, Lu and Garrido [8] explored the periodic NHPP model with a Beta-shaped intensity function.

The precision of claim count estimation is a key to running the insurance business successfully. In this study, we will present an estimation approach to non-life insurance claim counts related to a specification of the two different claim counting processes, i.e., HPP, and NHPP with a bell-shaped intensity function, through a simulation study. Our purpose is to estimate the parameters in the non-life insurance claim counting process. The parameters in the insurance claim counting process, intensity function $(\lambda(t))$ in terms of mean value function $\Lambda(t)=\int_{0}^{t} \lambda(u) \mathrm{d} u$, makes a complicated distribution function of insurance claim counts. An estimating function, such as the zero mean martingale (ZMM), is used here as a procedure of parameter estimation of an insurance claim counts model, and the parameters of model intensity are estimated by the MLE method.

\section{A Definition of the Non-Life Insurance Claim Counting Process}

We define the insurance claim counting process $N(t)=\#\left\{i \geq 1: T_{i} \leq t\right\} ; t \geq 0$, and the insurance claim counts which have occurred in the time interval $(0, t]$ where $T_{n}=W_{1}+\cdots+W_{n} ; n \geq 1$ is a claim arrival time and $W_{i}$ is independent and identically distributed (iid) Exponential with the parameter $\lambda\left(w_{i}\right)$, called the intensity rate, $N=\{N(t) ; t \geq 0\}$ is a counting process which is non-decreasing, $N(t)$ can be written as

$$
N(t)=\int_{0}^{t} \mathrm{~d} N(u) \text { where } \mathrm{d} N(t) \text { is an increment of } N
$$

in a small fraction period. The Poisson distribution is often considered as a common distribution modeling of insurance claim counts, and our main interest in the process of insurance claim counts is the Poisson process, i.e., HPP, and NHPP with the bell-shaped intensity function. This interest lies in the intensity rate, in which the insurance claim counts occur, and whether these change over time. In an HPP, the intensity rate is constant for a given time, and the process is called an NHPP, if it changes as a function of time [2,5,9].

On a probability space $\left(\Omega, \mathfrak{T}, P_{\Lambda}\right), N(t)$ is Poisson distributed with the parameter $\Lambda(t)=\int_{0}^{t} \lambda(u) \mathrm{d} u$, with a mean value function $E(N(t))=\Lambda(t)$. As $\{\lambda(t)=\alpha(t) \cdot k(t) ; t \geq 0\}$ is called the multiplicative intensity, where $\alpha(t)$ and $k(t)$ are defined as the intensity rate and the exposure risk, respectively. We con- sider $N$ as a non-decreasing right continuous step function 0 at time $t=0$ and jumps of size 1 , and

$$
P_{\Lambda}=\operatorname{Pr}\{N(t)=n\}=\frac{\Lambda(t)^{n} \exp (-\Lambda(t))}{n !} ; n=0,1,2, \cdots
$$

and $\operatorname{Pr}\{\mathrm{d} N(t)=1\}=\lambda(t) \mathrm{d} t=E\{\mathrm{~d} N(t)\}$.

In this study we consider the insurance claim counting process which are the HPP with $\lambda(t)=\lambda$, a constant intensity, and the NHPP with a bell-shaped intensity function as an initial season, $s=0$ [7],

$$
\lambda(t)=\frac{\lambda^{*} \exp \left\{-\frac{1}{2 \sigma^{2}}\left(t-\frac{1}{2}\right)^{2}\right\}}{\left(\Phi\left(\frac{1}{2 \sigma}\right)-\Phi\left(-\frac{1}{2 \sigma}\right)\right) \sigma \sqrt{2 \pi}}
$$

where $\lambda^{*}$ (an average number of claims over a period) and $\sigma$ are the parameters, $\lambda^{*}, \sigma>0$.

\section{Parameter Estimation in the Non-Life Insurance Claim Counting Process}

In this section, we introduce the methods which are useful for parameter estimation in the non-life insurance claim counting process, including the estimating function, the martingale method, and the MLE.

\subsection{Estimating Function}

On a probability space $\left(\Omega, \mathfrak{I}, P_{\Lambda}\right)$, where $\Lambda \in \Delta, \Delta$ is an open interval on the real line, $P_{\Lambda}=p(N(t) ; \Lambda)$. Suppose that the observation $N(t)=n$, the estimating functions, $g(N(t) ; \Lambda)$, are functions of $N(t)$ and the parameter $\Lambda$. By solving $g(N(t) ; \Lambda)=0$, a so-called estimating equation, an estimate of $\Lambda$ is obtained. Then $g(N(t) ; \Lambda)$ is an unbiased estimating function if $E[g(N(t) ; \Lambda)]=0$ for all $\Lambda \in \Delta \quad[10]$.

In this study, the estimating function for parameter estimation in the insurance claim counting process is provided by the martingale method.

\subsection{The Martingale Method}

The martingales are random processes relating to time. On a probability space $(\Omega, \mathfrak{T}, P)$, we suppose the increasing family $\wp=\left\{\wp_{t} ; t \geq 0\right\}$, a filtration or history $\delta_{t}$, which is the available data at the time $t$. The process $M=\{M(t) ; t \geq 0\}$ is a martingale with respect to $\wp$ if $E\{M(t)\}<\infty$ exist, and $E\left\{M(t+s) \mid \wp_{t}\right\}=M(t)$ for all $s \geq 0$. As a result of the properties of the martingale, $E\{M(t)\}=E\{M(0)\}$ for all $t \geq 0$, then $E\{M(0)\}=0$ for a ZMM [11,12].

This study of the martingale method is useful for constructing an estimating function for a parameter estima- 
tion in the insurance claim counting process. The process takes place over a small time interval $(t, t+\mathrm{d} t]$, $E\left\{\mathrm{~d} N(t) \mid \wp_{t-}\right\}=\lambda(t) \mathrm{d} t$ and as a result of the meaning of martingale property, the martingale can be written as

$$
\mathrm{d} N(t)-\lambda(t) \mathrm{d} t=\mathrm{d} M(t)
$$

which is a martingale-difference. Then, the following martingale is

$$
\int_{0}^{t}\{\mathrm{~d} N(u)-\lambda(u) \mathrm{d} u\}=\int_{0}^{t} \mathrm{~d} M(u),
$$

or it is rewritten in the form of $N(t)-\Lambda(t)=M(t)$, is a ZMM. Based on ZMM, we obtain

$$
E\{M(t)\}=0=E\{N(t)-\Lambda(t)\} .
$$

Thus, $N(t)-\Lambda(t)=0$ is an estimating equation for the parameter estimation in the insurance claim counting process. Also, as a result of the parameter estimate in the process, this can be interpreted as an $N(t)$ estimate or, in other words, $\Lambda(t)$ is called the compensator of $N(t)$, and this estimate is useful for predicting the times of occurrence of insurance claim counts [12]. We can depict the systematic part of the process of insurance claim counts, $N(t)$, related to its compensator, $\Lambda(t)$, and the associated martingale $N(t)-\Lambda(t)=M(t)$ in Figures 1(a) and (b), respectively, based on a sample of 15 independent random times of claims occurrence in the NHPP with an intensity of

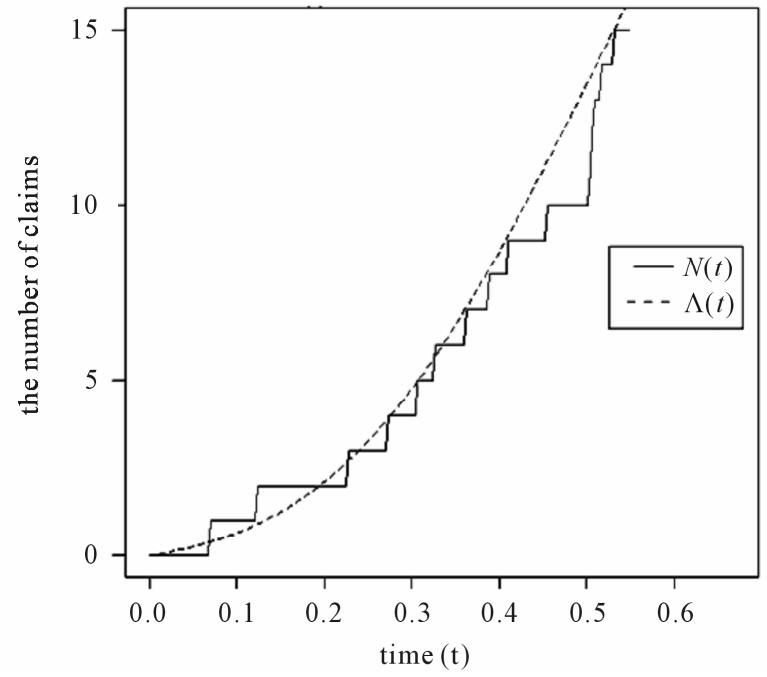

(a)

$$
\lambda(t)=48 \exp \left\{-10\left(t-\frac{1}{2}\right)^{2}\right\} .
$$

\subsection{A Maximum Likelihood Estimation of the Model Intensity}

In order to get the estimate of the compensator of $N(t)$, $\hat{\Lambda}(t)$, on the modeling of the non-life insurance claim counting process, both the HPP and NHPP, the parameters of the intensity function are estimated by the MLE method. Given $N(t)=n$, we suppose that $t_{1}, t_{2}, t_{3}, \cdots$, $t_{N(t)}$ are the arrival times of the claims in the time interval $(0, t]$ with a cumulative distribution function (a general order statistics model) $F(t)=1-\exp (-\Lambda(t))$. The likelihood function $[5,13]$ is given by

$$
l(\boldsymbol{\theta} ; \xi)=\prod_{i=1}^{n} \lambda\left(t_{i}\right) \exp \left(-\Lambda\left(t_{n}\right)\right)
$$

where $\boldsymbol{\theta}$ is a vector of the parameters of the model intensity, $\xi$ denotes the set of arrival times, the intensity $\lambda(t)$ in the HPP is $\lambda$ as $\boldsymbol{\theta}$ and the intensity $\lambda(t)$ in the NHPP is given in Equation (1) as $\boldsymbol{\theta}=\left(\lambda^{*}, \sigma\right)$. The estimate of $\boldsymbol{\theta}$ can be simply found if we take the logarithm of the likelihood function and we seek a value of $\boldsymbol{\theta}$ that maximizes the $\log$ likelihood function. The following parameter estimate of the HPP model is:

$\hat{\boldsymbol{\theta}}=\hat{\lambda}=\frac{n}{t_{N(t)=n}}$. For the NHPP, the calculation of the

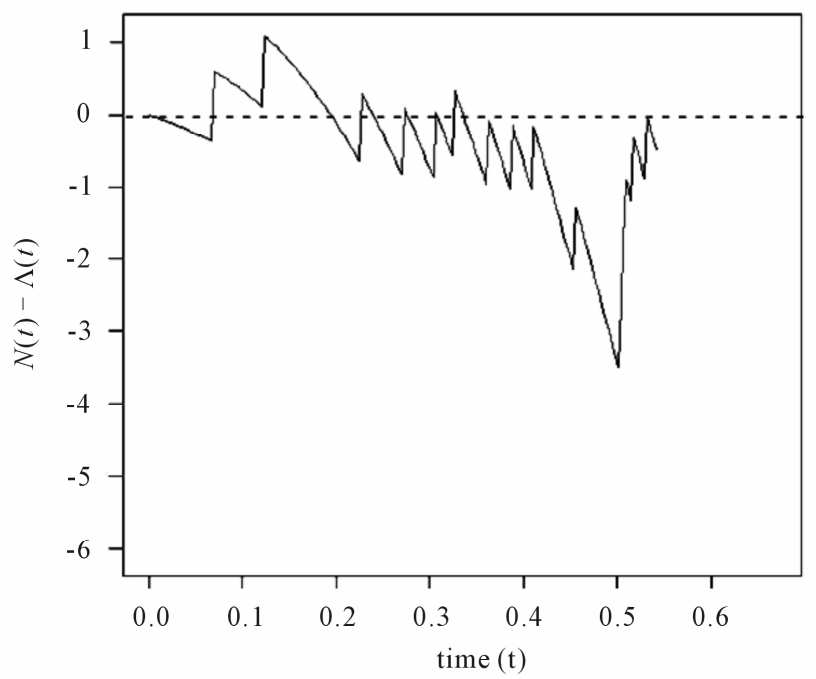

(b)

Figure 1. In a sample of 15 independent random times of claims occurrence with the intensity $\lambda(t)=48 \exp \left\{-10\left(t-\frac{1}{2}\right)^{2}\right\}$,

(a) Non-life insurance claim counting process $N(t)$ related to its compensator $\Lambda(t)$; and (b) Martingale

$M(t)=N(t)-\Lambda(t)$. 
MLE estimator of the model intensity, which is a complicated system of equations, requires an iterative procedure, i.e. the Newton-Raphson algorithm, to solve these equations [2].

\section{Simulation Study}

In this study, a simulation study is used to investigate how the observation of the non-life insurance claim counting process can be used to estimate its model parameter, i.e. intensity $\lambda(t)$ or in term $\Lambda(t)$, using the estimating function provided by the martingale method with ZMM. In particular, the HPP of the insurance claim counts, with $\lambda(t)=\lambda$ as a constant intensity and the NHPP of the insurance claim counts, with $\lambda(t)$ given in Equation (1) as a bell-shaped intensity, we must first consider the simulation study of the HPP of the insurance claim counts in the time interval $(0, t]$ in which the observation involves the claim arrival times, $t_{1}, t_{2}, t_{3}, \cdots$, $t_{N(t)}$, generated by applying an exponential law with the intensity $\lambda$, i.e. with a mean $1 / \lambda$ as $\lambda=0.1$ and 10 . The second simulation study of the insurance claim counting process, in which we consider the NHPP with a bell-shaped intensity function, or as the general form of mean value function

$$
\Lambda(t)=[t] \lambda^{*}+\lambda\left\{\Phi\left(\frac{t-[t]-0.5}{\sigma}\right)-\Phi\left(-\frac{1}{2 \sigma}\right)\right\}
$$

$t>0$, where [-] is the greatest integer function, the claim arrival times, $t_{1}, t_{2}, t_{3}, \cdots, t_{N(t)}$, can be simulated by using the mean value function $\Lambda(t)$ as a claim arrival time of the HPP with mean one [7]. It implies that $E_{1}, E_{2}, E_{3}, \cdots, E_{n}$ are independent and exponentially distributed with mean one, where $E_{i}=\Lambda\left(t_{i}\right)-\Lambda\left(t_{i-1}\right)$, for all $i=1,2,3, \cdots, n$. So, in this study, the $n^{\text {th }}$ claim arrival time, $t_{N(t)=n}$, is generated by

$$
t_{N(t)=n}=\Lambda^{-1}\left(E_{1}+E_{2}+E_{3}+\cdots+E_{n}\right)
$$

where $\Lambda^{-1}(\alpha)=\left[\frac{\alpha}{\lambda^{*}}\right]+\sigma \Phi^{-1}\{D\}+0.5$ is the invertible function of $\Lambda(t), D=\frac{\alpha-\lambda^{*}\left[\frac{\alpha}{\lambda^{*}}\right]}{\lambda^{*}} \Phi_{D}+\Phi\left(-\frac{1}{2 \sigma}\right)$, $\Phi_{D}=\Phi\left(\frac{1}{2 \sigma}\right)-\Phi\left(-\frac{1}{2 \sigma}\right)$ and $\Phi^{-1}$ is the quantile function of a standard normal distribution, with $\lambda^{*}=0.1$, $\sigma=0.25, \quad \lambda^{*}=0.1, \quad \sigma=5, \lambda^{*}=10, \sigma=0.25$ and $\lambda^{*}=10, \sigma=5$.

In this simulation study of both the HPP and the NHPP of the insurance claim counts in the time interval $(0, t]$, the number of observations, $N(t)=n$, is composed of 5 , 10, 15 and 20. The HPP and the NHPP are carried out with 5000 sample paths. In each sample path, the parameter estimate of the model intensity is computed using the MLE method and the estimating function, such as the ZMM which is used to estimate the parameter $\Lambda(t)$ in the process (or the compensator $\Lambda(t)$ of $N(t)$ ), i.e. fitting the compensator estimate $\hat{\Lambda}(t)$ to $N(t)$. Also, the mean squared error (MSE) is provided to measure things, fitting $\hat{\Lambda}(t)$ to $N(t)$ as the following form,

$$
\text { MSE }=\sum_{i=1}^{p} \frac{\int_{0}^{t}\left(\hat{\Lambda}_{i}(u)-N_{i}(u)\right)^{2} \mathrm{~d} u}{p},
$$

where $p$ denotes the number of sample paths. Notice that the MSE of the compensator estimate $\hat{\Lambda}(t)$ of $N(t)$ for both processes, as shown in Tables $\mathbf{1}$ and $\mathbf{2}$, depends on the parameters of the model intensity as the following details, for the HPP with a constant intensity $\lambda=0.1$, a small intensity rate, and the MSE of the compensator estimate $\hat{\Lambda}(t)$ of $N(t)$ increases exponentially as the number of observations increases. On the other hand, the same process with $\lambda=10$, the MSE of the compensator estimate $\hat{\Lambda}(t)$ of $N(t)$ decreases exponentially, while the observation number increases until the observed 15 times of claims occurrence, and then the MSE value begins to increase as the observation number increases. For the NHPP with a bell-shaped intensity, the

Table 1. MSE of the compensator estimate $\hat{\Lambda}(t)$ of $N(t)$ in the HPP of non-life insurance claim counts.

\begin{tabular}{cccccc}
\hline$\lambda$ & $N(t)$ & MSE & $\lambda$ & $N(t)$ & MSE \\
\hline \multirow{2}{*}{0.1} & 5 & 1.003785 & & 5 & 11.90220 \\
& 10 & 1.834148 & & 10 & 5.776900 \\
& 15 & 2.665881 & 10 & 15 & 4.904133 \\
& 20 & 3.528792 & & 20 & 5.012547 \\
\hline
\end{tabular}

Table 2. MSE of the compensator estimate $\hat{\Lambda}(t)$ of $N(t)$ in the NHPP of non-life insurance claim counts.

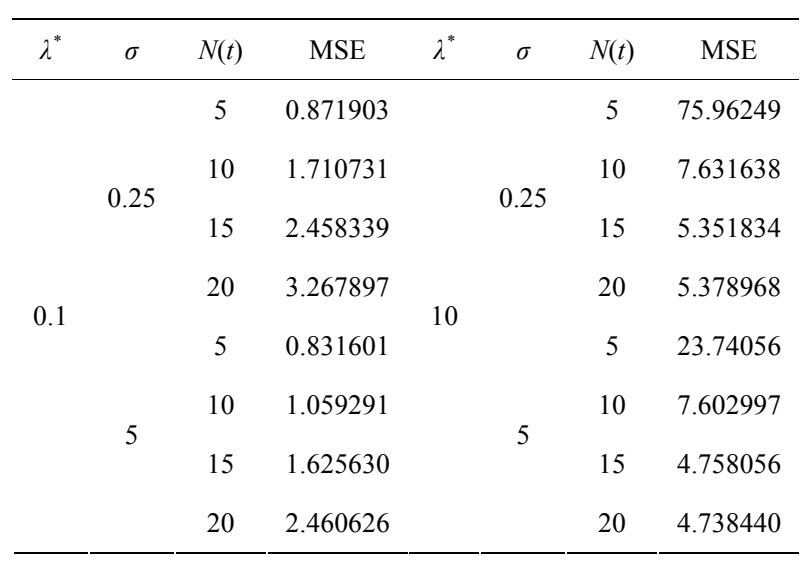


parameters of its model intensity $\lambda^{*}=0.1, \sigma=0.25$ and $\lambda^{*}=0.1, \sigma=5$ (a small average number of a claims over a period), the MSE of the compensator estimate $\hat{\Lambda}(t)$ of $N(t)$ increases as the observation number increases. When we consider the NHPP between the parameters of the model intensity $\lambda^{*}=0.1, \sigma=0.25$ and $\lambda^{*}=0.1, \sigma=5$, we found that in the process with the parameters of the model intensity $\lambda^{*}=0.1, \sigma=5$, the MSE of the compensator estimate $\hat{\Lambda}(t)$ of $N(t)$ is much lower. In the same NHPP with the parameters of model intensity $\lambda^{*}=10, \sigma=0.25$, the MSE of the compensator estimate $\hat{\Lambda}(t)$ of $N(t)$ decreases while its observation number increases until the observed 15 times of claims occurrence, and then its MSE values begins to increase as the observation number increases. The MSE of the compensator estimate $\hat{\Lambda}(t)$ of $N(t)$ in the same NHPP with the parameters $\lambda^{*}=10, \sigma=5$ decreases exponentially as its observation number increases. When we consider the NHPP between the parameters of the model intensity $\lambda^{*}=10, \sigma=0.25$ and $\lambda^{*}=10$, $\sigma=5$, we found that in the process with the parameters

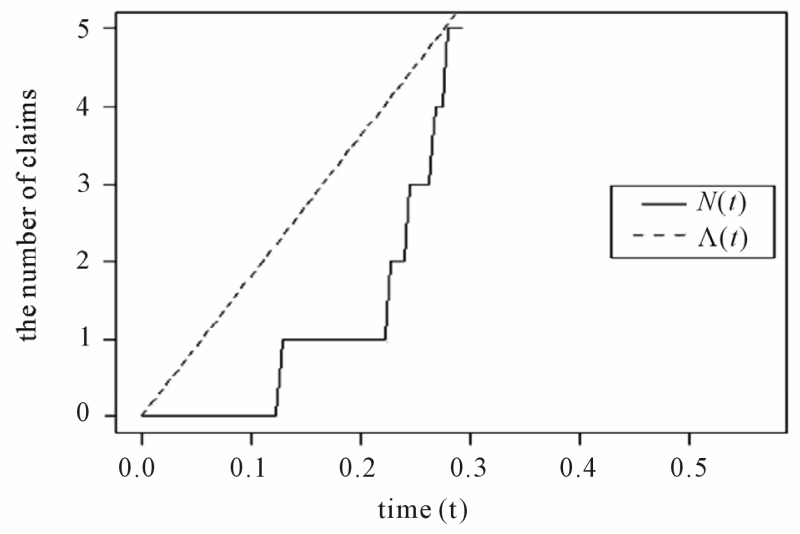

(a)

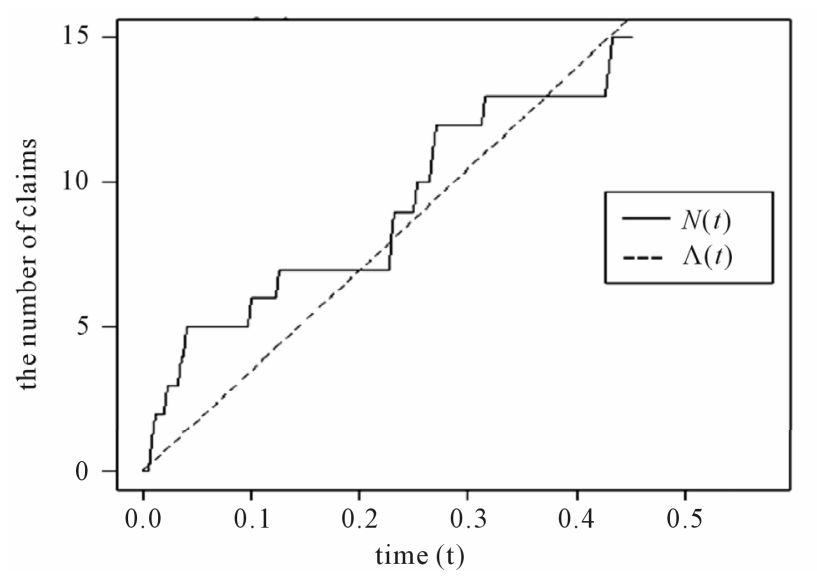

(c) of the model intensity $\lambda^{*}=10, \sigma=5$, the MSE of the compensator estimate $\hat{\Lambda}(t)$ of $N(t)$ is much lower than the other one.

Some examples in these situations of both the HPP and the NHPP with a bell-shaped intensity of non-life insurance claim counts based on a sample of 5, 10, 15 and 20 times of claims occurrence are illustrated in Figures 2 and 3, including the $N(t)$ and its compensator $\Lambda(t)$. Figure 2 shows a sample path of the HPP with a constant intensity $\lambda=10$. The $N(t)$ and its compensator $\Lambda(t)$ are characterized by the intensity $\lambda$, i.e. $\lambda=10$, the compensator $\Lambda(t)$ fits well with $N(t)$, as the observation number is 15 and 20 (slightly larger than the intensity $\lambda=10)$. Similarly, the $N(t)$ and its compensator $\Lambda(t)$ in the NHPP are characterized by the parameters of the model bell-shaped intensity $\lambda^{*}=10$, $\sigma=5$ in Figure 3. The compensator $\Lambda(t)$ fits with $N(t)$, as the observation number is 15 and 20 (slightly larger than the parameter of model intensity $\left.\lambda^{*}=10\right)$. Figure 4 illustrates a sample path of NHPP, and we can see the difference with the compensator estimate which

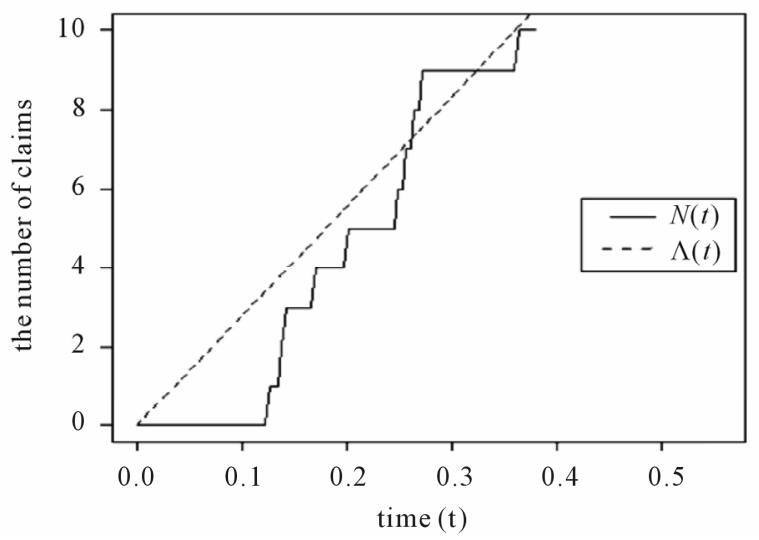

(b)

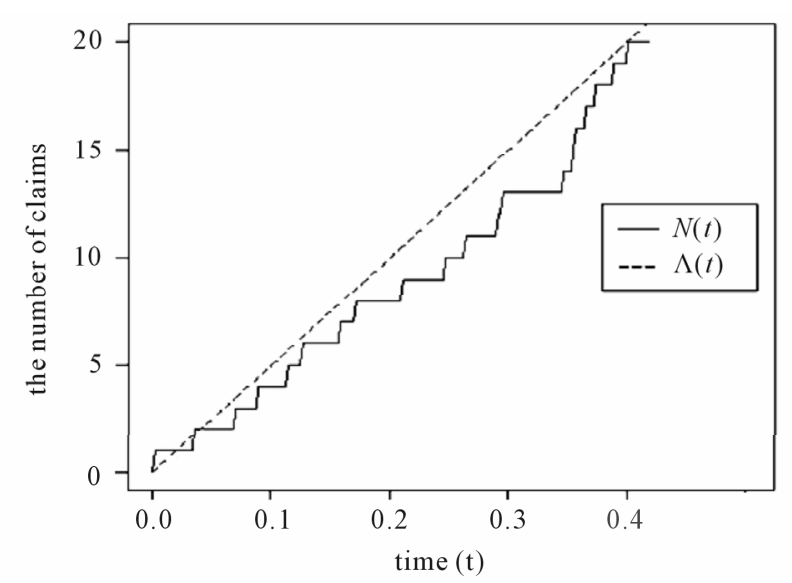

(d)

Figure 2. $N(t)$ and its compensator $\Lambda(t)$ in the HPP with the intensity $\lambda=10$ based on a sample of (a) 5 claims; (b) 10 claims; (c) 15 claims; and (d) 20 claims. 


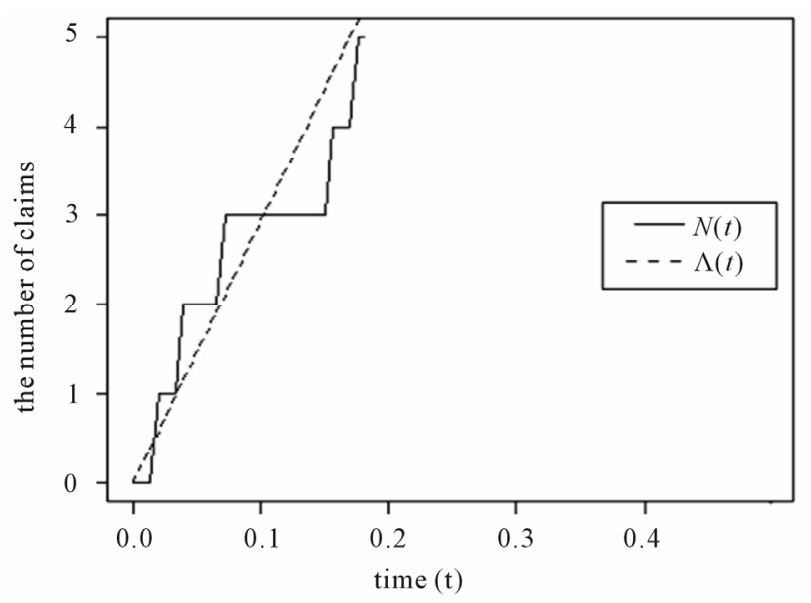

(a)

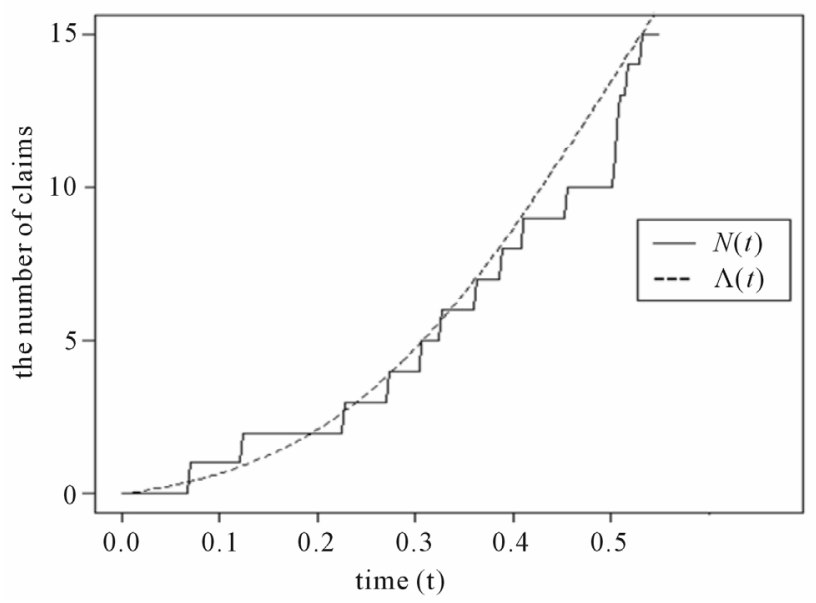

(c)

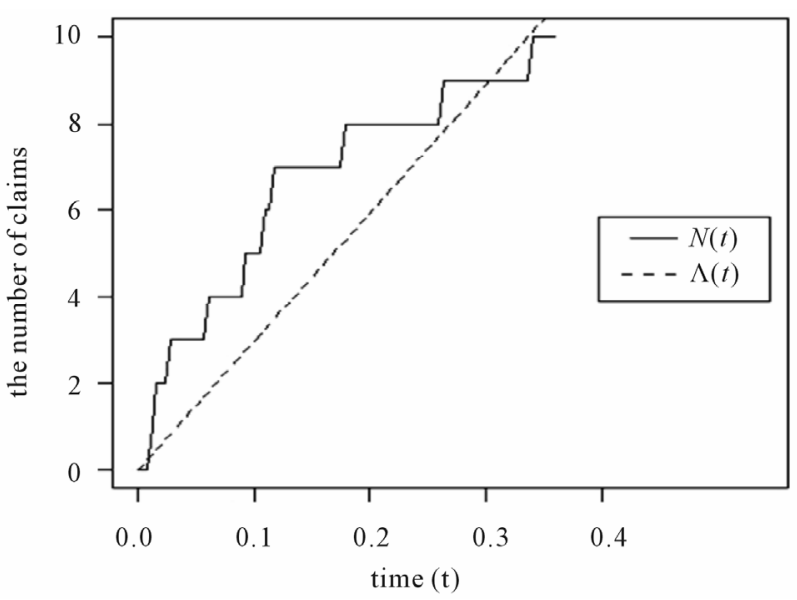

(b)

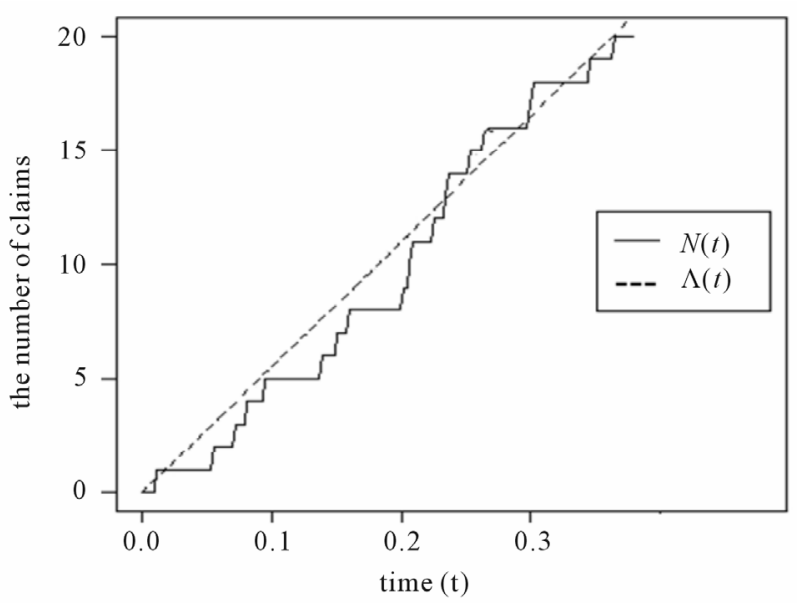

(d)

Figure 3. $N(t)$ and its compensator $\Lambda(t)$ in the NHPP with the parameters of a bell-shaped intensity $\lambda^{*}=10, \sigma=5$ based on a sample of (a) 5 claims; (b) 10 claims; (c) 15 claims; and (d) 20 claims.

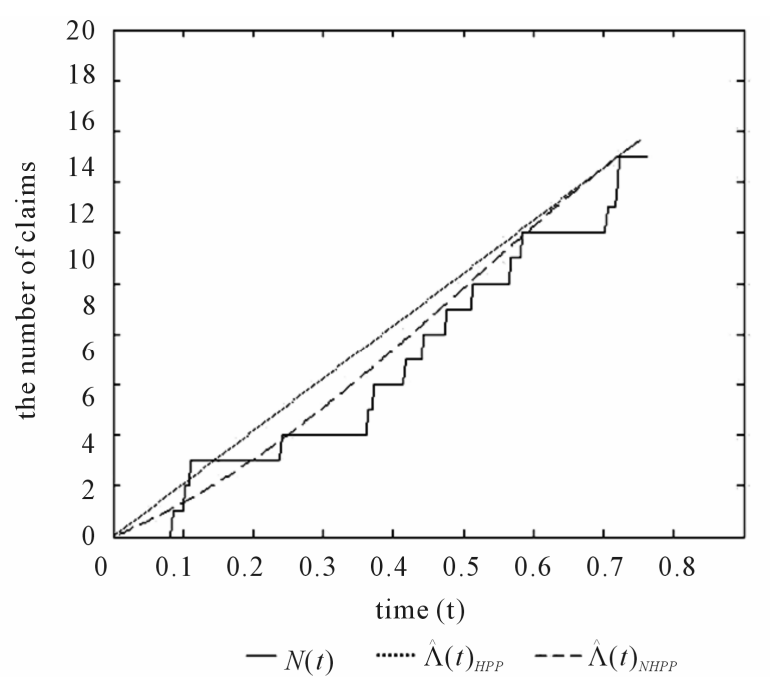

Figure 4. $N(t), \hat{\Lambda}(t)_{\mathrm{HPP}}$, and $\hat{\Lambda}(t)_{\mathrm{NHPP}}$ in the NHPP of non-life insurance claim counts. uses the estimation method of NHPP with a period timedependent intensity, $\hat{\Lambda}(t)_{\mathrm{NHPP}}$, fits well with $N(t)$ by $\mathrm{MSE}=1.48$ shown with a dashed line and the $\mathrm{MSE}=5.35$ along 5000 sample paths. It is notable that if, instead, the compensator estimate misspecification $\hat{\Lambda}(t)_{\mathrm{NHPP}}$ is calculated more easily by using the estimation method of HPP with a constant intensity, and the compensator estimate misspecification $\hat{\Lambda}(t)_{\mathrm{HPP}}$ fluctuates a lot from $N(t)$, shown on the dotted line in Figure 4. The MSE of fitting the compensator estimate misspecification $\hat{\Lambda}(t)_{\mathrm{HPP}}$ to $N(t)$ is 2.72, and the MSE $=5.53$ along 5000 sample paths are larger than the fitting of the compensator estimate $\hat{\Lambda}(t)_{\mathrm{NHPP}}$ to $N(t)$.

\section{Conclusion}

This simulation study of the non-life insurance claim counting process, of both the HPP and the NHPP with a bell-shaped intensity, demonstrates that the fitting of the 
compensator estimate $\hat{\Lambda}(t)$ to $N(t)$ in the time interval $(0, t]$ depends on the parameters of model intensity as in the following details, firstly, regarding the HPP with a small intensity rate, with almost no claim occurrences, while the number of observations is very small, the compensator estimate $\hat{\Lambda}(t)$ is a good fit to $N(t)$ with less of a MSE. In the same process with a constant intensity rate, the claims occurrence rate, when the number of observations is slightly larger than the constant intensity, the MSE of the compensator estimate $\hat{\Lambda}(t)$ of $N(t)$ is much less. Secondly, as regards to the NHPP with the parameters of the model intensity, a $\lambda^{*}$ has a very small average number of claims over a period, almost no claim occurrences over a period, and any $\sigma$, as the number of observations is very small, the compensator estimate $\hat{\Lambda}(t)$ is a good fit to $N(t)$ with less of a MSE. Using the same process with the parameters of the model intensity, with an average number of claims over a period $\lambda^{*}$ is no less than one and any $\sigma$, while the number of observations is slightly larger than the value of $\lambda^{*}$, the MSE of the compensator estimate $\hat{\Lambda}(t)$ of $N(t)$ is much less. Some examples of the situations in the simulation study are also depicted by a sample path relating $N(t)$ and its compensator $\Lambda(t)$. Furthermore, the result of the compensator estimate misspecification $\hat{\Lambda}(t)$ of $N(t)$ is illustrated by a sample path of the NHPP so that the MSE of fitting the compensator estimate misspecification $\hat{\Lambda}(t)$ to $N(t)$ is much larger than the fitting of the compensator $\Lambda(t)$ to $N(t)$.

\section{REFERENCES}

[1] S. A. Klugman, H. H. Panjer and G. E. Willmot, "Loss Models from Data to Decisions," 3rd Edition, John Wiley \& Sons, Hoboken, 2008.

[2] M. Denuit, X. Maréchal, S. Pitrebois and J. F. Walhin, “Ac- tuarial Modelling of Claim Counts," John Wiley \& Sons, Hoboken, 2007. doi:10.1002/9780470517420

[3] H. Bühlmann, "Introduction Report Experience Rating and Credibility," ASTIN Bulletin, Vol. 4, No. 3, 1967, pp. 199-207.

[4] H. Bühlmann, "Credibility Procedures," Proceedings of the Sixth Berkeley Symposium on Mathematical Statistics and Probability, University of California Press, Berkeley, Vol. 1, 1972, pp. 515-525.

[5] T. Mikosch, "Non-Life Insurance Mathematics," 2nd Edition, Springer-Verlag, Berlin, 2009. doi:10.1007/978-3-540-88233-6

[6] M. Matsui and T. Mikosch, "Prediction in a Poisson Cluster Model," Journal of Applied Probability, Vol. 47, No. 2, 2010, pp. 350-366. doi:10.1239/jap/1276784896

[7] M. Morales, "On a Surplus Process under a Periodic Environment: A Simulation Approach," North American Actuarial Journal, Vol. 8, No. 2, 2004, pp. 76-87.

[8] Y. Lu and J. Garrido, "On Double Periodic Non-Homogeneous Poisson Processes," Bulletin of the Swiss Association of Actuaries Swiss Association of Actuaries-Bern, Bern, 2004, pp. 195-212.

[9] S. M. Ross, "Introduction to Probability Models," 5th Edition, Academic Press, Inc., San Diego, 1993.

[10] P. Mukhopadhyay, "An Introduction to Estimating Functions," Alpha Science International Ltd., Harrow, 2004.

[11] P. K. Andersen, O. Borgan, R. D. Gill and N. Keiding, "Statistical Models Based on Counting Processes," SpringerVerlag, New York, 1993.

[12] P. Yip, "Estimating the Number of Error in a System Using a Martingale Approach," IEEE Transactions on Reliability, Vol. 44, No. 2, 1995. pp. 322-326. doi:10.1109/24.387389

[13] J. E. R. Cid and J. A. Achcar, "Bayesian Inference for Nonhomogeneous Poisson Processed in Software Reliability Models Assuming Nonmonotonic Intensity Functions," Computational Statistics \& Data Analysis, Vol. 32, No. 2, 1999, pp.147-159. doi:10.1016/S0167-9473(99)00028-6 